\title{
Microscopic origin of the non-Gaussian behavior of dynamic structure factors of glassy matter
}

\author{
C. Cabrillo, ${ }^{1}$ M. A. González, ${ }^{2}$ G. J. Cuello, ${ }^{2}$ F. J. Bermejo, ${ }^{1}$ M. L. Saboungi, ${ }^{3,4}$ and D. L. Price ${ }^{1,3,4}$ \\ ${ }^{1}$ Consejo Superior de Investigaciones Científicas, Instituto de Estructura de la Materia, Serrano 123, E-28006 Madrid, Spain \\ and Department of Electricity and Electronics, University of the Basque Country, P.O. Box 644, Bilbao 48080, Spain \\ ${ }^{2}$ Institut Laue Langevin, Boîte Postale 156x, F-38042 Grenoble Cedex 9, France \\ ${ }^{3}$ Argonne National Laboratory, Argonne, Illinois 60439, USA \\ ${ }^{4}$ C.N.R.S., University of Orleans, 45071 Orleans Cedex 2, France
}

(Received 12 February 2003; revised manuscript received 10 December 2003; published 12 April 2004)

\begin{abstract}
We consider the Lamb-Mössbauer factors corresponding to the structure factors of a material (ethyl alcohol) showing two glassy phases, one of those being an orientationally disordered crystal. The deviations from the idealized Gaussian behavior expected for an isotropic-harmonic vibrator within the amorphous phase and disordered crystal are found to be remarkably close. Such proximity enables us to take advantage of the crystal symmetry to gain access to specific details of the effective interparticle potential. Once this is done the treatment is extended to include the fully amorphous material. The results depict atomic motions within the glassy matrices as significantly anisotropic and highly anharmonic and thus provide a way to understand the microscopic origin of phenomena considered as fingerprints of glassy dynamics.
\end{abstract}

DOI: 10.1103/PhysRevB.69.134202

PACS number(s): 63.50.+x, 61.43.-j

\section{INTRODUCTION}

Most glasses show thermal expansion coefficients that typically are one order of magnitude larger than those of their parent crystals. ${ }^{1}$ This taken together with the well documented anomalies in sound velocity and attenuation at low temperatures ${ }^{2}$ depicts the thermal properties of these states of matter as fundamentally different from their fully ordered crystalline forms. On such grounds one would expect to find signatures of such distinctive behaviors in a variety of dynamical properties accessible to experiment. Those concerning the microscopic realm such as the temperature and wavevector dependences of atomic motions are known to be sensitive to departures from the behavior followed by simple model systems such as perfect crystals at low temperatures where particle motions are driven by harmonic potentials. Such signatures of nonideal behavior can nowadays be calculated for simple solids such as the condensed rare gases. In fact, several decades of work on the explicit calculation of thermodynamic properties and spectra for realistic models of anharmonic crystals, ${ }^{3}$ has achieved remarkable accuracy in predicting thermal and dynamical properties of crystals close to melting if high-order terms in the Van Hove ordering parameter are included in the calculations. While those methods can, in principle, be applied to any condensed system whether ordered or not, most treatments dealing with amorphous and disordered-crystalline matter are usually of phenomenological nature.

In recent times we have carried out studies on the volumic dependence of the vibrational spectrum of a material showing a rich polymorphism. ${ }^{4}$ The system just referred to concerns the amorphous, orientationally disordered/rotatorphase (i.e., where the average position of the molecular centers-of-mass occupies the nodes of a bcc lattice) and fully ordered crystal forms of ethanol. ${ }^{5-9}$ The concurrent study of the several solid phases has allowed to quantify the effects of anharmonicity and disorder within the fully ordered, orientationally disordered, and fully amorphous phases. ${ }^{4}$ Here we pursue such studies now focusing onto the effects on nonideal behavior on a basic dynamical property such as the asymptotic form of the $G_{s}(r, t)$, space-time self-correlation function for $t \rightarrow \infty$ that is experimentally accessible by means of measurements of the Lamb-Mössbauer factor. The relevance of such studies stems from the use of departures of such quantity from its Gaussian form,

$$
G_{s}(r, \infty)=\left(\frac{3}{4 \pi\left\langle u^{2}\right\rangle}\right)^{3 / 2} \exp \left(-3 r^{2} / 4\left\langle u^{2}\right\rangle\right),
$$

as measures of deviation from ideality in glass-forming liquids. ${ }^{10}$ Here $\left\langle u^{2}\right\rangle$ stands for the particle mean-squared displacement, which characterizes the thermal motion of a particle subjected to an isotropic harmonic potential. Such particle displacements within a liquid arise from the action of both stochastic forces leading to mass diffusion and rapid oscillations due to interparticle vibrations. As one approaches the glass transition from above, the former kind of motions are expected to decrease in relative importance with respect to those of vibrational nature up until a point where mass diffusion becomes slow enough to be observable within usual observation windows.

Deviations from the harmonic form for $G_{s}(r, t)$ measured by experimental means ${ }^{10-14}$ or calculations ${ }^{15,16}$ are often interpreted as fingerprints for regions within the disordered material where groups of atoms move with mean-squared displacements far larger than the average thermal value. More into specifics, the experimental evidence for nonideal behaviors is usually quantified by a strong nonideal behavior of the appropriate response function. In our particular case this translates into "non-Gaussian" features in the dynamic structure factors in neutron-scattering experiments. ${ }^{10}$ For a given temperature $T$, such departures refer to the wavevector dependence of the incoherent elastic scattering intensity $I_{s}(Q, \omega=0, T)=f_{s}(Q, T)=\exp [-2 W(Q, T)]$ that equals $\exp \left(-\alpha Q^{2}\right)$ for a vibrating isotropic body subjected to harmonic restoring forces having an average mean-square dis- 
placement (m.s.d.) given by $\alpha=\frac{1}{3}\left\langle u^{2}\right\rangle$. Here $f_{s}(Q, T)$ stands for the time integral taken over $F_{s}(Q, t)$, the fourier transform of $G_{s}(r, t)$ for a given temperature. In such a case deviations from a Gaussian distribution of atomic displacements will give additional terms in the $Q$ dependence of $W(Q, T)$ such as,

$$
2 W(Q, T)=\frac{1}{3}\left\langle u^{2}\right\rangle Q^{2}-\frac{1}{24}\left[P\left\langle u^{4}\right\rangle Q^{4}-\cdots\right]
$$

where the second term contains contributions up to fourth order in the displacements whose explicit form for the coefficient $P$ depends upon details of higher-order terms in the interaction potential. A convenient parameter to measure departures from the Gaussian shape is given by the ratio of fourth-to-second moments of the particle displacements,

$$
A_{n g}(t)=\frac{3\left\langle\Delta r^{4}(t)\right\rangle}{5\left\langle\Delta r^{2}(t)\right\rangle^{2}}-1,
$$

which vanishes for Gaussian motion.

Preliminary results from computer simulations carried out for a realistic model of ethanol within the glass and liquid states described in Ref. 5 that are displayed in Fig. 1 serve to illustrate the extent in time and space as well as the temperature dependence of deviations from ideal behavior.

A glance at Fig. 1 shows that even within the normal liquid range (i.e., for $T>200 \mathrm{~K}$ purely Gaussian behavior is found at times of the order of tenths of a picosecond as well as at long times when particle motions conform to hydrodynamic diffusion. Moreover, such non-Gaussian behavior is seen to be strongly temperature dependent. As temperature lowers, the strength of the deviation as well as the lapse of time taken to enter the diffusive regime become increasingly large. In fact, for temperatures below $160 \mathrm{~K}$ it takes a few nanoseconds to regain the Gaussian regime. The maximum of the $A_{n g}(t)$ curves $A_{n g}^{\max }$ is known to appear when the contribution to the mean-square displacements arising from diffusive motions becomes comparable to that due to rapid motions of vibrational nature. ${ }^{15}$ The quantity $A_{n g}^{\max } t^{*}$ giving the value of $A_{n g}(t)$ at its maximum multiplied by the time elapsed to reach it, is expected to show a relatively welldefined feature at some temperature signaling the transition into a strong non-Gaussian regime. The middle frame of Fig. 1 depicts such a quantity that shows a change of slope for temperatures within $120 \mathrm{~K}-150 \mathrm{~K}$.

As a spatial counterpart, the lower frame of Fig. 1 shows the particle displacement probability as measured from $4 \pi r^{2} G_{s}(r, t)$ for the molecular centers of mass for the latter temperature. The relevant feature there concerns the rather different regimes observed for times of the order of tenths of picoseconds where particle excursions cover distances of about $0.5 \AA$ and the subsequent entrance within a regime of sluggish motions. In fact, from there we see that for times between 1 and 1000 ps particle displacements are confined within a sphere of about $2 \AA$ of radius.

From what has just been written it is clear that the signatures of non-Gaussian behavior will show strong space, time, and temperature dependencies. The question of how the deviations from Gaussianity observed in the liquid relate to
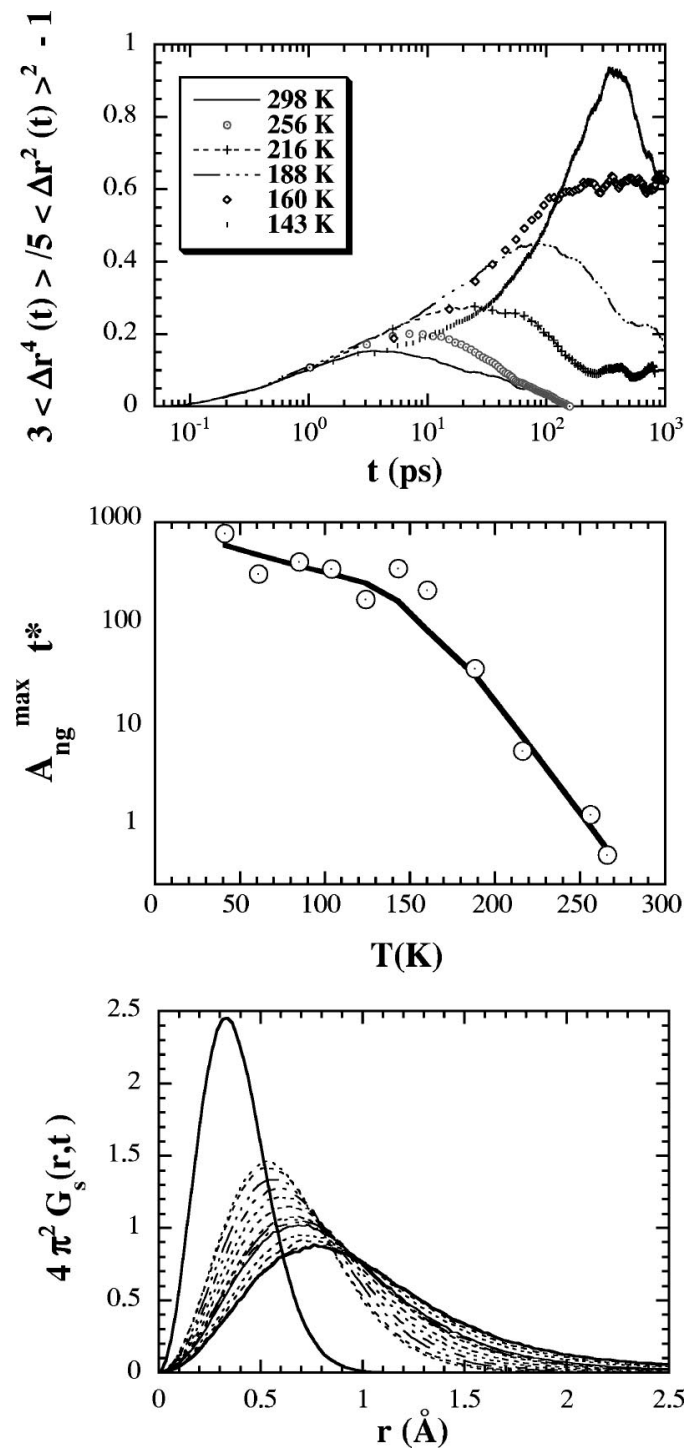

FIG. 1. The upper frame depicts results for the non-Gaussian parameter $A_{n g}(t)$ as calculated from computer molecular dynamics simulations for the set of molecular centers of mass for temperatures given in the inset. The middle frame shows the dependence with temperature of the quantity $A_{n g}^{\max } t^{*}$ (see text). The solid line is a smoothing spline shown as a guide to the eye. The lower frame shows the probability that a particle moves a distance $r$ in a time interval $t$ as measured by $4 \pi r^{2} G_{s}(r, t)$. The leftmost curve is taken for $t=0.2 \mathrm{ps}$. The observation times for the rest, from left to right, are $1,2,5,10,20,40,120,160,200,400,800$, and 1000 ps. Calculations here correspond to $T=160 \mathrm{~K}$.

those found for the deep glass phase remains open. As a matter of fact, within the glass state observations of groups of atoms executing motions with amplitudes well above the average thermal value are known from computer simulations ${ }^{17}$ as well as direct experimental observations. ${ }^{11,14}$ A unified approach should therefore comprise both the spatial (or wave vector) and temperature dependencies of such deviations. Here we aim to provide such a tool on the basis of our own measurements of departures from ideal behavior spanning temperatures from the deep glass up to the normal liquid range on a relatively simple system such as ethanol 
which is studied both experimentally by neutron scattering as well as by computer simulation. Our aim is to track down the origin of such behaviors to details of the interparticle interaction potential. More into details, our study concerns a sample that can be easily prepared in its fully amorphous, liquid, and orientationally disordered forms. Its simple molecular structure $\mathrm{CH}_{3} \mathrm{CH}_{2} \mathrm{OH}$ and the possibility of studying the deviations from Gaussianity within the rotator-phase crystal paves up the way to tackle the fully amorphous sample since theoretical results for the non-Gaussian behavior of cubic crystals are known in detail. In addition, the sample here studied presents some advantages over more complex materials since it is free from effects of static local microscopic anisotropy common to more complex materials such as polymers. Furthermore, its anharmonic properties have been quantified in detail in terms of Grüneisen parameters ${ }^{4}$ and this will surely help to understand the deviations from Gaussianity at least at a semiquantitative level.

In what follows we will first describe the results from a set of neutron-scattering experiments carried out to validate the simulation results. The latter are then used to gain access to quantities not directly amenable to experiment such as those concerning the molecular centers of mass. Computation of such quantities allows to carry out an analysis couched in terms of an effective interparticle potential that includes terms up to a fourth order in the particle displacement.

\section{EXPERIMENTAL MEASUREMENTS}

The experimental data were measured on the IN6 spectrometer at the Institute Laue Langevin, Grenoble (France) for an extended set of temperatures. The measurements are complementary to those already reported ${ }^{4,7-9}$ that were focused on comparisons between spectra and dynamics for the various condensed phases as well as transformations between them. ${ }^{18}$

Fully deuterated ethanol samples were used in order to ascertain the sample state (amorphous or disordered crystal) through diffraction measurements. The quantities to be derived from experiment are the temperature-dependent $W(Q, T)$ exponents of the Lamb-Mössbauer factors (i.e., the Debye-Waller factor for an incoherent-scattering signal) that are related to the measured dynamic structure factors $S(Q, \omega)$. Their calculation was performed through the computation of the quantity $f_{s}(Q, T)=\ln [S(Q, \omega=0, T)-S(Q, T)$ $+1]=2 W(Q, T)$, where $S(Q, \omega=0, T)$ is the elastic structure factor calculated by integration of the elastic peak over limits $(0.15 \mathrm{meV})$ commensurable with the achieved instrument resolution and $S(Q, T)=\int d \omega S(Q, \omega, T)$.

The departure from Gaussian behavior as far as its spatial dependence is concerned is usually quantified ${ }^{10,16}$ by a dimensionless parameter $A_{n g}=2 c_{4} / c_{2}^{2}$, where $c_{2}$ and $c_{4}$ are coefficients of the $Q^{2}$ and $Q^{4}$ terms needed to account for the observed decays of $f_{s}(Q, T)$. This measure of nonGaussianity is a convenient tool to depict the extent of the spatial dependence of the deviation from ideality at a glance, although a more complete picture will require the analysis of

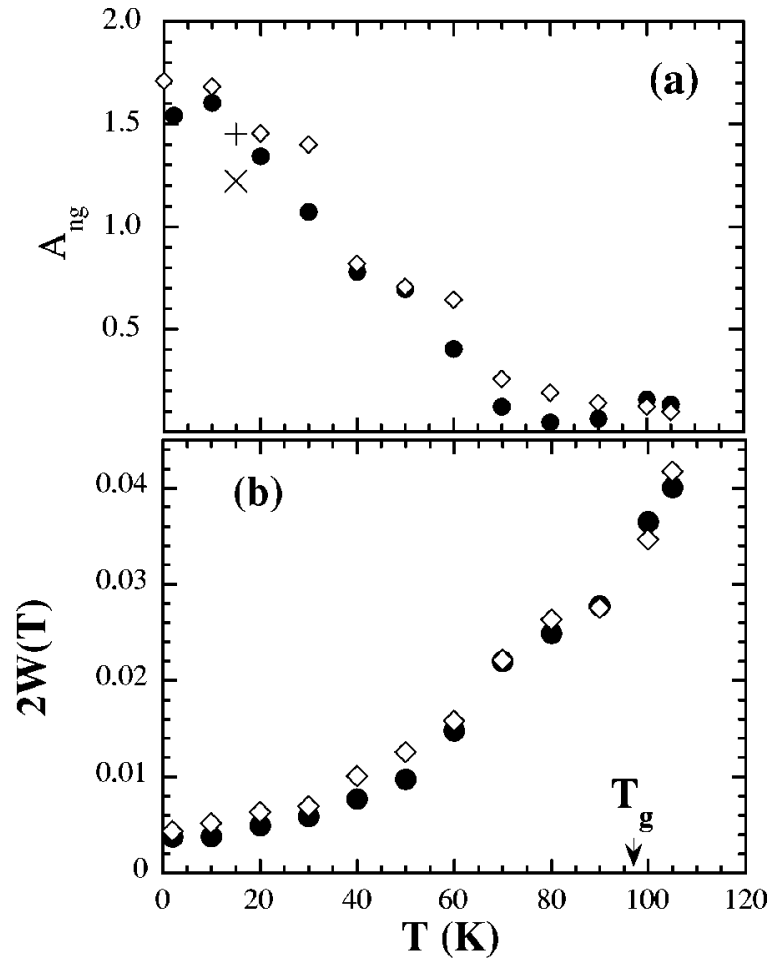

FIG. 2. (a) Deviation of $I_{s}(Q, \omega=0)$ from Gaussian behavior derived from experimental measurements as measured by the parameters $A_{n g}$. Open symbols stand for the glass (and supercooled liquid) and filled symbols for the orientationally disordered cubic crystals. Crosses denote data measured using the MARI spectrometer for glass $(+)$ and orientationally disordered crystal $(x)$. (b) Experimental estimates for the quantities $2 W(T)$ entering the exponent of the Lamb-Mössbauer factor. The symbol $T_{g}$ marks the temperature where the calorimetric transitions take place.

the temperature dependence of both $c_{2}$ and $c_{4}$ coefficients as discussed below.

Having in mind the proviso of our limited range of wave vectors $\left(Q\right.$ range $\approx 0.2-3.0 \AA^{-1}$ ) accessible to our measurement, we have calculated the experimental $A_{0}$ parameters for the disordered crystals and glass and which are shown in Fig. 2. A test on the significance of the quantities just referred to is provided by data measured over a far larger range of momentum-transfers using the MARI chopper instrument at the ISIS pulsed neutron source. Such data ${ }^{7}$ also shown in Fig. 2 for a single temperature reveal a reasonably good agreement with the present results. This measure of nonGaussianity for the glass/liquid and disordered crystal phases shows both ordering states to be rather close, a result that comes into line with the thermodynamic and dynamical features examined so far. ${ }^{25,26}$

The values for $A_{n g}$ shown in Fig. 2 become large as we enter the glass phase from above. The behavior shown by experiment is in at least a qualitative agreement with simulation data shown in Fig. 1. The strong rise of such parameter with decreasing temperature is due to the distinct dependency of temperature shown by the fourth-order term compared to its second-order counterpart and detailed considerations of this particular are deferred to latter sections. The $A_{n g}$ parameters for the glass and rotator-phase crystal show 


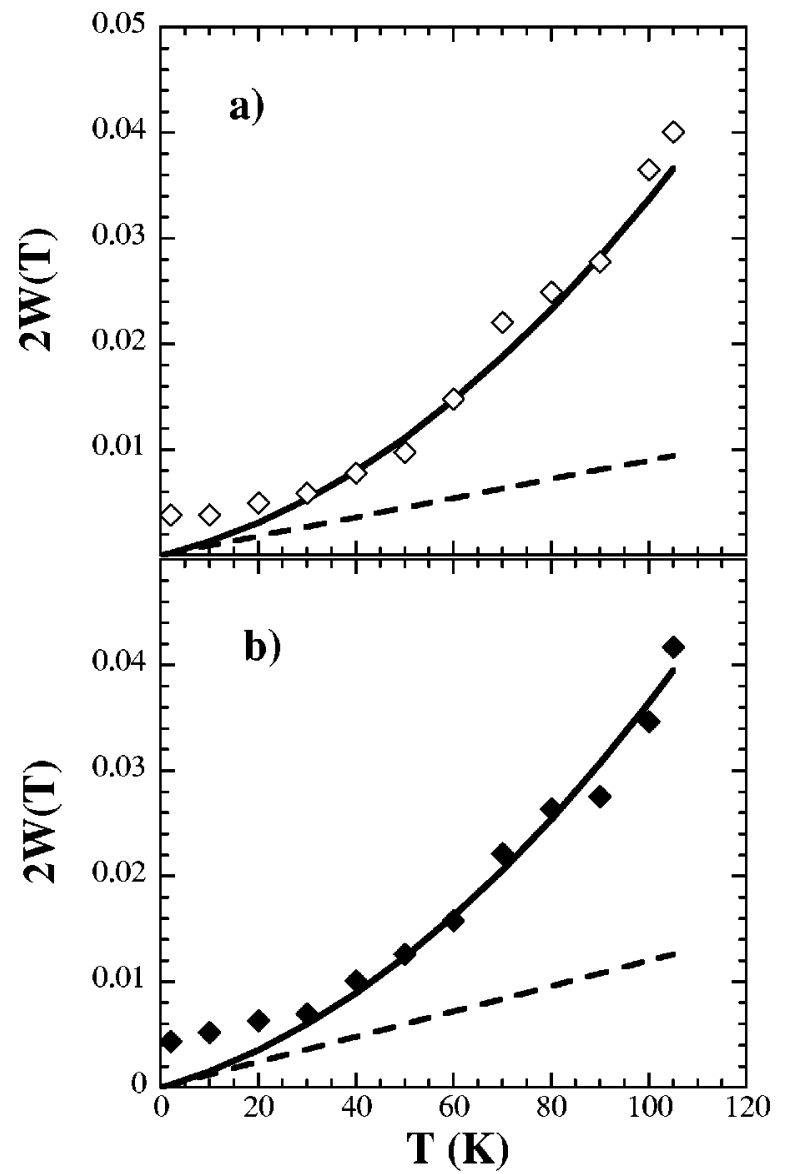

FIG. 3. Temperature dependence of the terms entering the exponent of the Lamb-Mössbauer factors. The upper frame depicts data for the glass/supercooled liquid and the lower frame those for the orientationally disordered/rotator-phase crystals, respectively. Symbols stand for experimental values, dashed lines are the results for the harmonic approximation calculated following steps given in the text, and solid lines are the result from the anharmonic approximation (see text).

far milder temperature dependencies once the glass $\rightarrow$ liquid and qrientationally disordered crystal (ODC) transitions cross into the supercooled liquid and rotator-phase crystal, respectively. This takes place upon crossing the respective thermodynamic glass-transitions centered at about $95 \mathrm{~K}$.

A first step towards a detailed study of the temperature dependence of non-Gaussian behavior is better done in terms of $W(Q, T)$. Furthermore, to simplify the analysis and to improve on the statistics of the experimental data an average over $Q$ wave vectors is performed so that we deal with a quantity $W(T)$ only dependent upon temperature.

Figure 3 displays the obtained results. As expected from the close similarity found for $A_{0}$ for both phases, data concerning $W(T)$ again show the same close behaviors. On the basis of such similitude we consider the disordered crystal as an adequate test ground to go deeper into the nature of the deviations from Gaussianity.

Data displayed in Fig. 3 show that $W(T)$ strongly departs from the expectation values calculated for purely harmonic behavior, i.e., $W(T) \propto T$. To quantify such a deviation we first extrapolate the value for $T=0$ and compare it to that calculated for a cubic crystal which reads as ${ }^{19}$

$$
2 W(0)=\frac{\hbar \overline{Q^{2}}}{2 M_{e f f}} \int_{0}^{\omega_{m} Z(\omega)} \frac{\omega}{\omega} d \omega,
$$

where $Z(\omega)$ is density of one-phonon states, $\omega_{m}$ the maximum phonon frequency, the horizontal bar denotes average over $Q$, and $M_{\text {eff }}$ is the effective mass of the scatterer as seen by the neutron probe. By fitting $W(T)$ to third order polynomials the values of the $W(0)$ 's were calculated by extrapolation to $T \rightarrow 0$. Making now recourse to the previous knowledge of the experimental value for the first negative frequency moment ${ }^{4}$ leads to estimates for $1 / M_{\text {eff }}$, the inverse of the effective mass, of $0.83 \pm 0.2$ for the glass and $0.66 \pm 0.2$ for the disordered crystal, both given in terms of the molecular mass.

For a cubic crystal, a purely harmonic dependence of $W(T)$ upon temperature should follow ${ }^{19,20}$

$$
2 W(T)=\frac{\hbar \overline{Q^{2}}}{2 M_{e f f}} K_{B} T \int_{0}^{\omega_{m}} \frac{Z(\omega)}{\omega^{2}} d \omega .
$$

Since $1 / M_{\text {eff }}$ is now set, the temperature dependence of $W(T)$ given by Eq. (5) can now be calculated using for this purpose our knowledge of the experimental value for the second negative moment given in Ref. 4. The result also shown in Fig. 3 serves to set a comparison with the experimental estimates for $W(T)$ which strongly deviate from the harmonic approximation. This is known to be caused by anharmonic effects ${ }^{4}$ that typically manifest themselves at mesoscopic scales where the material behaves as an elastic continuum (i.e., typically over distances of a thousand angstrom or so) in the marked temperature dependence of the fractional change in sound velocity $\delta \omega / \omega$ usually measured by Brillouin or mechanical spectroscopies. ${ }^{4}$

The first correction to the harmonic approximation includes the effects of thermal expansion. Its relative strength is evaluated from data concerning volume expansivity $\chi$ and macroscopic Grüneisen parameters $\gamma_{G} \cdot{ }^{4}$ It amounts to renormalize the harmonic value for the m.s.d. by $\left(1+2 \gamma_{G} \chi T\right)$, which makes $W(T)$ to display a temperature dependence slightly nonlinear. In real figures calculated for a reference temperature of $80 \mathrm{~K}$, this correction amounts to multiply the harmonic values of the m.s.d. by 1.1 and 1.2 for the glass and the ODC, respectively. However, as data shown in Fig. 3 reveal, such correction term cannot account for the large value of $W(T)$ in any of both phases, making a more complete anharmonic treatment necessary to reproduce the data. The details of such a calculation are given in more explicit terms in the coming sections. Here we only point to a comparison of results from such a treatment with experimental data, both sets shown in Fig. 3. Notice that such a comparison is made fitting $1 / M_{\text {eff }}$ as the only adjustable parameter. Moreover, the values we get that are $0.83 \pm 0.02$ for the glass and $0.63 \pm 0.01$ times the inverse molecular mass for the disordered crystal are in very good agreement with the estimates given above obtained from $W(0)$. 


\section{COMPUTER SIMULATIONS}

The most relevant details concerning the interaction potentials, algorithms, and integration steps for the molecular dynamics simulations have already been given. 5 ,9 The employed model considers the molecule as "semiflexible" with a low-lying internal degree of freedom constituted by the internal molecular rotation about the $\mathrm{C}-\mathrm{O}$ bond. Intermolecular forces are felt on four sites located at the methyl, methylene, oxygen and hydroxyl groups and comprise both Lennard-Jones, and electrostatic interactions. In addition, the internal rotation about the C-O bond is described by a Fourier series with coefficients chosen to give reasonable agreement with experimental data.

A further study on the effects of a complete molecular force-field $^{21}$ (including explicitly all atoms within the molecule) on the calculated properties was also carried out. For obvious reasons the calculations were restricted to short times ( 40 ps of equilibration followed by 40 ps of production runs) compared to the ones reported here (several nanoseconds). The calculated quantities which were under particular scrutiny were the density of vibrational states, mean-squared displacements, and those characterizing the reorientational dynamics. The density of vibrational states, which is the quantity most affected by explicit inclusion of all the intramolecular vibrations, shows important changes with respect to that calculated for the four-interaction site model. These are, however, distributed over frequencies above 25 $\mathrm{meV}$, while the shape of the low-frequency band centered at about $6 \mathrm{meV}$ is basically unaltered. The mean-square displacements calculated by the all-atom model show systematic differences with respect to the four-site representation, the latter being somewhat larger than the former. This effect becomes more important as the temperature is increased. In fact, below some $220 \mathrm{~K}$ there is very little difference between the $\left\langle\Delta r^{2}(t)\right\rangle$ curves for both models. Finally, the same qualification applies to the calculated orientational correlation functions which also tend to decay somewhat faster than those calculated for the four-site model.

The finite-size effects were also evaluated by means of computations on a far larger ensemble (1728 versus 216 molecules). Calculations were performed using large equilibration times (1.2 ns) and simulations of $1 \mathrm{~ns}$ followed. There, similar findings to those reported for vitreous silica are found, ${ }^{22}$ that is, differences in $\left\langle\Delta r^{2}(t)\right\rangle$ become amplified as the temperature is lowered. In consequence, the data reported here concern the larger sample size.

The simulations were carried out either starting from liquid configurations that once equilibrated are quenched to form the glass or from a bcc array where the lattice nodes correspond to centers of mass of randomly oriented ethanol molecules which is then allowed to relax. ${ }^{5}$ The temperature ranges corresponding to the transitions glass $\rightarrow$ liquid and orientationally disordered $\rightarrow$ rotator phases were determined by changes of slope of the curves showing the temperature dependence of the density as well as from deviations shown by plots of translational and rotational diffusion coefficient from the Vogel-Fulcher-Tamann law. Both transitions take place within a temperature interval of $100 \mathrm{~K}-135 \mathrm{~K}$, that is,

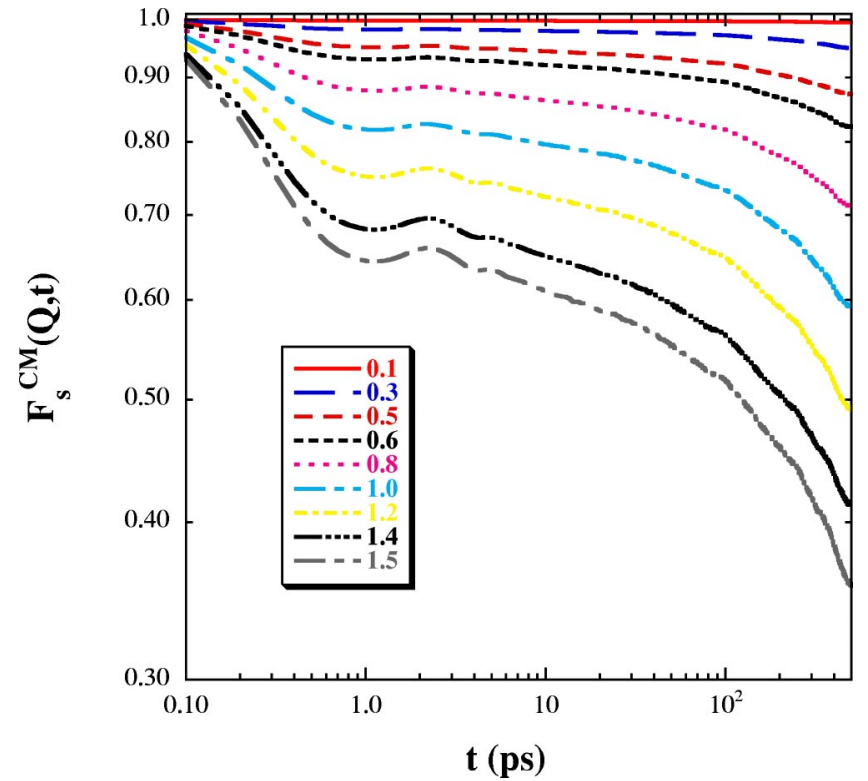

FIG. 4. Time and wave-vector dependencies of $F_{s}^{C M}(Q, t)$ for supercooled states as calculated by molecular dynamics simulations, for $T=120 \mathrm{~K}$. The inset lists the set of wave-vector values expressed in reciprocal angstrom units.

some $20 \mathrm{~K}$ above experiment. Complete arrest of all stochastic dynamic quantities is found for temperatures below 100 $\mathrm{K}$. On the other hand, the normal equilibrium liquid range is achieved for temeperatures somewhat above $170 \mathrm{~K}$.

In what follows, we will focus our discussion on quantities calculated for the molecular centers of mass such as the $F_{s}^{C M}(Q, t)$ intermediate scattering functions defined as

$$
F_{s}^{C M}(Q, t)=\frac{1}{N} \sum_{i}\left\langle\exp \left[-i Q r_{i}^{C M}(0)\right] \exp \left[i Q r_{i}^{C M}(t)\right]\right\rangle,
$$

where the sum runs over all molecules within the sample having centers of mass located at $r_{i}^{C M}(t)$ at time $t$. The rationale for choosing these quantities instead of their atomic correlates stands for the greater simplicity of handling (i.e., rotational motions are only felt via the translation-rotation coupling terms). Also, such simplifying representation of the molecular dynamics enables us to employ for its analysis already existing theoretical frameworks developed for monoatomic cubic crystals. ${ }^{3,20}$ An example of the calculated quantities is shown in Fig. 4. There we show a set of intermediate scattering functions corresponding to a deeply supercooled state (i.e., a state still showing a finite value of $10^{-12} \mathrm{~cm}^{2} \mathrm{~s}^{-1}$ for the mass-diffusion coefficient). The curves shown there exhibit an initial fast decay up to some 2 ps or so, followed by a more sluggish relaxation that can be adequately described in terms of a Kohlrausch-WilliamsWatts law as described in Ref. 5 and is usually identified with the $\alpha$ relaxation that appears as the strongest peak in dielectric relaxation measurements. Below $\approx 90 \mathrm{~K}$ there is no evidence for a further relaxation beyond the fast initial decay. From the calculated quantities we have evaluated the function $f_{s}^{C M}(Q, T)$ from where the $Q$ and temperature de- 


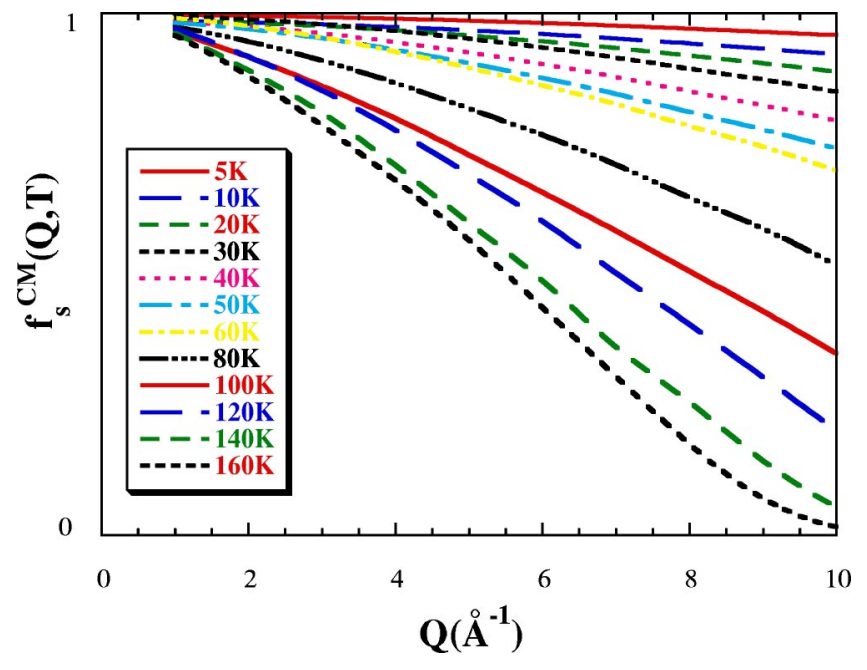

FIG. 5. Wave-vector dependence of the quantity $F_{s}(Q, T)$ for the disordered crystals as calculated by molecular dynamics for temperatures given in the inset.

pendence of its parameters can be evaluated, as referred below. Figure 5 displays a set of $f_{s}^{C M}(Q, T)$ functions for the disordered/rotator-phase crystals. These are obtained from the simulated self-intermediate scattering functions by time integration in a $800 \mathrm{ps}$ time window. From the graphs plotted there we witness a deviation from quadratic (harmonic) behavior in $Q$ that grows stronger as the temperature is increased.

\section{NON-GAUSSIAN BEHAVIOR: QUANTITATIVE ASSESSMENT}

We now take advantage of the close behaviors shown by glass and disordered crystal states. As our point of departure we start by considering an analytically tractable object which is an array of molecular centers of mass (CM) corresponding to our bec disordered crystal as calculated from the simulations. Such an array can be treated using a theory developed for cubic crystals. ${ }^{23}$ It predicts that the $W(Q, T)$ terms entering the Lamb-Mössbauer factors should follow, ${ }^{23}$

$$
\begin{aligned}
2 W(Q, T)= & Q^{2} \frac{k_{B} T}{\alpha_{0}}\left[1+\left(2 \gamma_{G} \chi-20 \frac{\gamma}{\alpha_{0}} \frac{k_{B}}{\alpha_{0}}\right) T\right] \\
& -Q^{4}\left(\frac{k_{B}}{\alpha_{0}}\right)^{3}\left(2 \frac{\gamma}{\alpha_{0}}+\frac{9}{80} \frac{\delta}{\alpha_{0}}\right) T^{3} .
\end{aligned}
$$

The equation written above gives the exponent for the Lamb-Mössbauer factor of an individual CM that experiences an effective potential $V(r)=(\alpha / 2) r^{2}+\gamma r^{4}+\delta\left[x^{4}\right.$ $\left.+y^{4}+z^{4}-(3 / 5) r^{4}\right]$ corresponding to Einstein-like oscillators with $x, y, z$ along the principal directions of the bcc lattice and $r$ is the displacement modulus. Thermal expansion effects are again accounted for on the grounds of the usual quasiharmonic approximation by renormalization of the three potential coefficients by $1 / \alpha \rightarrow\left(1+2 \gamma_{G} \chi T\right) / \alpha .{ }^{23}$ The $9 / 80$ factor in Eq. (7) results from the orientational average of the anisotropic contribution coming from the $\delta$ term, and

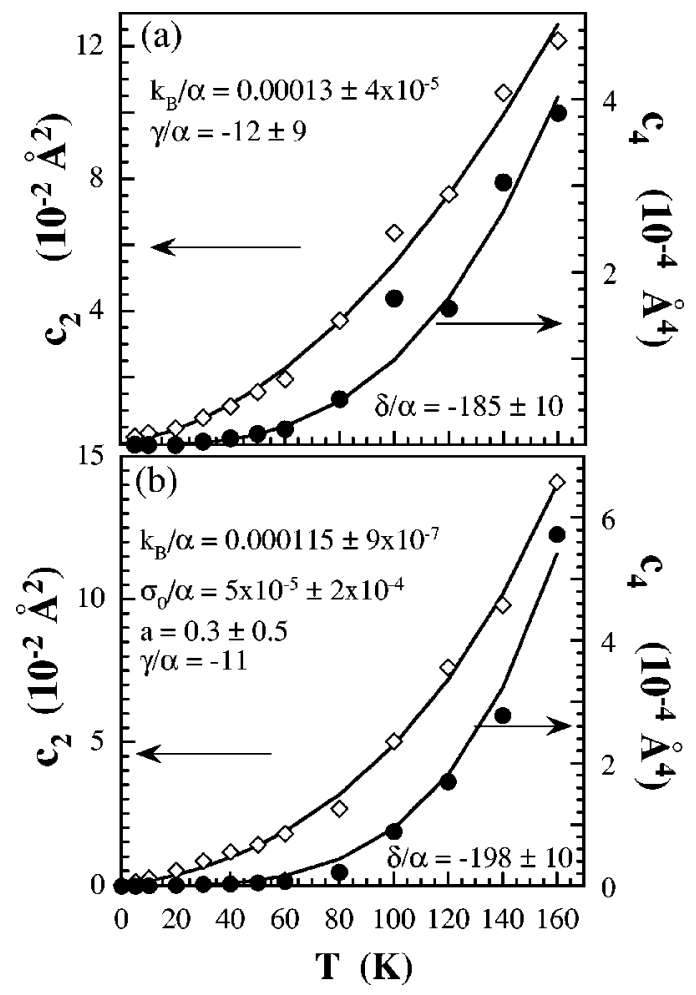

FIG. 6. Temperature dependence of the quadratic $\left(c_{2}\right.$, open symbols) and quartic ( $c_{4}$, filled symbols) coefficients needed to account for the wave-vector dependence of $2 W(Q, T)$ as calculated for the molecular centers of mass by means of the computer simulations. The upper frame shows simulation data for the disordered crystals and the lower frame shows equivalent data for the glass. Solid lines are fits to Eqs. (7) and (8), respectively.

is needed to account for the polycrystalline nature of our sample. Formally the same expression is obtained within a perturbation theory of nonlinear phonon interactions. ${ }^{20}$

Figure 6(a) shows the temperature dependence of both the $Q^{2}\left(c_{2}\right)$ and $Q^{4}$ terms $\left(c_{4}\right)$ needed to describe the wavevector behavior of $f_{s}^{C M}(Q, T)$ together with fits using Eq. (7) with $2 \gamma_{G} \chi$ taken from experiment. Notice that fitting $c_{4}$ involves only one parameter, since $c_{4} \propto T^{3}$ is assumed. From here one gets estimates for the potential coefficients that yield values of $\gamma / \alpha=-12$ and $\delta / \alpha=-185$. This shows that $V(r)$ is highly anharmonic with a quartic term two orders of magnitude larger than the harmonic part. A cursory glance at such figures deems such large quartic term as too large and calls for an assessment on the range of validity of Eq. (7).

Equation (7) is obtained through canonical averaging of $\exp (i \vec{Q} \cdot \vec{r})$ using the above written expression for $V(r)$. To enable an analytical integration, the canonical distribution $\exp \left[-V(r) / k_{B} T\right]$ is approximated by $\exp \left[-\alpha r^{2} /\left(2 k_{B} T\right)\right][1$ $\left.\left.-\gamma r^{4} /\left(k_{B} T\right)-\delta\left(x^{4}+y^{4}+z^{4}\right)-(3 / 5) r^{4}\right) /\left(k_{B} T\right)\right]$. This means that the harmonic part is treated exactly and this yields the Gaussian prefactor. The condition of validity for the present approximation tells that the equation should be valid for any value of $r$ provided that the anharmonic terms are much smaller than their squares within a standard deviation of the Gaussian part. In more precise words, such condition means $r<\sqrt{k_{B} T / \alpha}$, and this is certainly the case for the parameters 
obtained here. This is understood on the basis of the superexponential decay of the Gaussian prefactor which compensates the polynomial growth of the anharmonic term. Physically this means that the linear contribution to the potential energy is dominant within a spatial range comparable to the size of the thermal cloud even if the anharmonic coefficients are larger than their harmonic counterpart. In consequence we have to bear in mind that the quartic term in Eq. (7) still is a correction to the quadratic one since it is cubic in the relevant parameter, that is, $k_{B} T / \alpha$.

The negative sign of the $\gamma$ coefficient corresponds to a potential that "softens" for large displacements, a feature which is central to some approaches to glassy dynamics such as that couched in terms of the "soft potential model" $(\mathrm{SPM}) .{ }^{24}$ In close parallelism "non-Gaussian" behavior arises there as a natural consequence of the action of a large quartic term in the potential which also has force constants that are randomly distributed.

Our result also shows that particle motions are strongly anisotropic due to the large value of $\delta / \gamma$. A positive value for such ratio (i.e., $\delta<0$ ) conforms to the symmetry of the bcc lattice. In particular, the present estimate of $\delta / \gamma=15.4$ corresponds to softening along the $\langle 100\rangle$ directions concomitant with hardening along the $\langle 111\rangle$ directions which is consistent with the repulsive effect of neighboring molecules. ${ }^{7}$

\section{A STEP FURTHER: THE FULLY DISORDERED SOLID}

We now show how to extend the result for an ensemble of molecular CM's devoid of the regularity imposed by the bcc lattice. Obviously, Eq. (7) cannot strictly be applied to a phase lacking cubic symmetry. Here, however, we will profit from the close similarity of the orientationally disordered crystal with respect to the glass since both show close densities, and some common features concerning the short-range order. ${ }^{6}$ In addition, as shown in Ref. 27 formation of the disordered crystal state from the glass/supercooled liquid is driven by a purely entropic term, which leads the system into a state of intermediate thermodynamic stability with respect to the fully ordered crystal. That is, such transformation basically involves no significant change in internal energy. On such grounds we make use of Eq. (7) as our point of departure in search for a more general expression.

To pursue an adequate representation of glass data we follow the steps of previous treatments couched in terms of soft potentials ${ }^{24}$ and allow the coefficients entering the $V(r)$ potential to be randomly distributed rather than being singlevalued quantities. This is done to account for the disordered structure, since all terms entering Eq. (7) are explicitly dependent upon the crystal-field symmetry. Our treatment thus requires to take an additional average over the parameters. To a first approximation, the only averages we need concern a few negative moments of the leading term $\alpha$. In turn, these moments are expanded around the mean value of $\alpha$ up to second order, i.e., the variance of $\alpha$. The final expressions for the quadratic and quartic coefficients read

$c_{2}=\widetilde{k}_{B} T\left(1+2 \gamma_{G} \chi T\right)\left[1+\tilde{\sigma}(T)^{2}\right]-20 \tilde{\gamma} \tilde{k}_{B}^{2} T^{2}\left[1+6 \tilde{\sigma}(T)^{2}\right]$,

$$
\begin{aligned}
c_{4}= & \frac{1}{8} \widetilde{k}_{B}^{2} T^{2}\left(1+2 \gamma_{G} \chi T\right)^{2} \widetilde{\sigma}(T)^{2} \\
& -\widetilde{k}_{B}^{3}\left(2 \tilde{\gamma}+\frac{9}{80} \widetilde{\delta}\right) T^{3}\left[1+10 \widetilde{\sigma}(T)^{2}\right],
\end{aligned}
$$

where the tilde denotes division by $\alpha$ with $\alpha, \gamma$, and $\delta$ now understood as mean values of the corresponding potential parameters, and $\sigma(T)$ stands for the width of the distribution of values for $\alpha$ before applying the quasiharmonic renormalization. Furthermore, all the potential coefficients entering Eq. (7) can be expected to be temperature dependent, specially under conditions where thermal expansion effects are deemed to be severe (i.e., not too far below melting). ${ }^{20,23}$ Here, we have found that in order to include such effects a renormalization of the width of the distribution for the leading parameter suffices. In fact, we find that the simplest possibility that is to assume a linear temperature dependence that is $\tilde{\sigma}(T)=\tilde{\sigma}_{0}(1+a T)$ is able to account for the current data. The temperature dependence of the width of the distribution can thus be viewed as resulting from an increase in size of the thermal cloud. The result can also be related to results cast in terms of average cage frequencies and its frequency spread that increase with increasing temperature, ${ }^{31}$ able to describe the dynamics of supercooled liquids.

Figure 6(b) shows the simulation data as well as the corresponding fits to Eqs. (8). The $c_{2}$ coefficient was fitted setting $\tilde{\gamma}$ to a fixed value to improve the convergence of the fit. Starting from $\tilde{\gamma}=12$, which was the value found for the crystal phase, a refined estimate gave $\tilde{\gamma}=11$ as the best value. The $c_{4}$ was then fitted taking the obtained parameter values for $c_{2}$ so that only $\widetilde{\delta}$ remains as a free parameter. The quality of the fits is again remarkable. Their self-consistency as well the closeness to the disordered-crystal results make us confident of having grasped the underlying physics.

To check the robustness of this approach, we have carried out a number of tests. First, Eq. (7) was found unable to fit the glass data. Next, we tried either fitting the glass data using the harmonic part of Eqs. (8) or to restrict the effect of disorder to the harmonic part, or even to change the prefactors of the variance terms. In all cases just referred to selfconsistency was not achieved nor unphysical values were obtained, also resulting in poor quality fits.

\section{CONNECTION WITH EXPERIMENT}

Turning now back to experimental data shown in Fig. 3, we are now in a position to carry out a quantitative analysis of the experimental $W(T)$ using for the purpose Eq. (7). Furthermore, to scrutinize the reliability of parameters obtained from simulations, we will now proceed leaving just only one free parameter to account for the small differences in effective masses found between simulation and experiment. This amounts to include within the equations describing $W(Q, T)$ the change $\widetilde{k}_{B} \rightarrow \widetilde{k}_{B} / M^{*}$. In other words, having set the values of the parameters entering the $V(r)$ potential with the aid of molecular simulations we now use such information to calculate the experimental estimates for $W(T)$. The result of such fits is shown in Fig. 3 and, as mentioned above, it yields $1 / M^{*}=0.83 \pm 0.02$ and $1 / M^{*}=0.63 \pm 0.01$ 


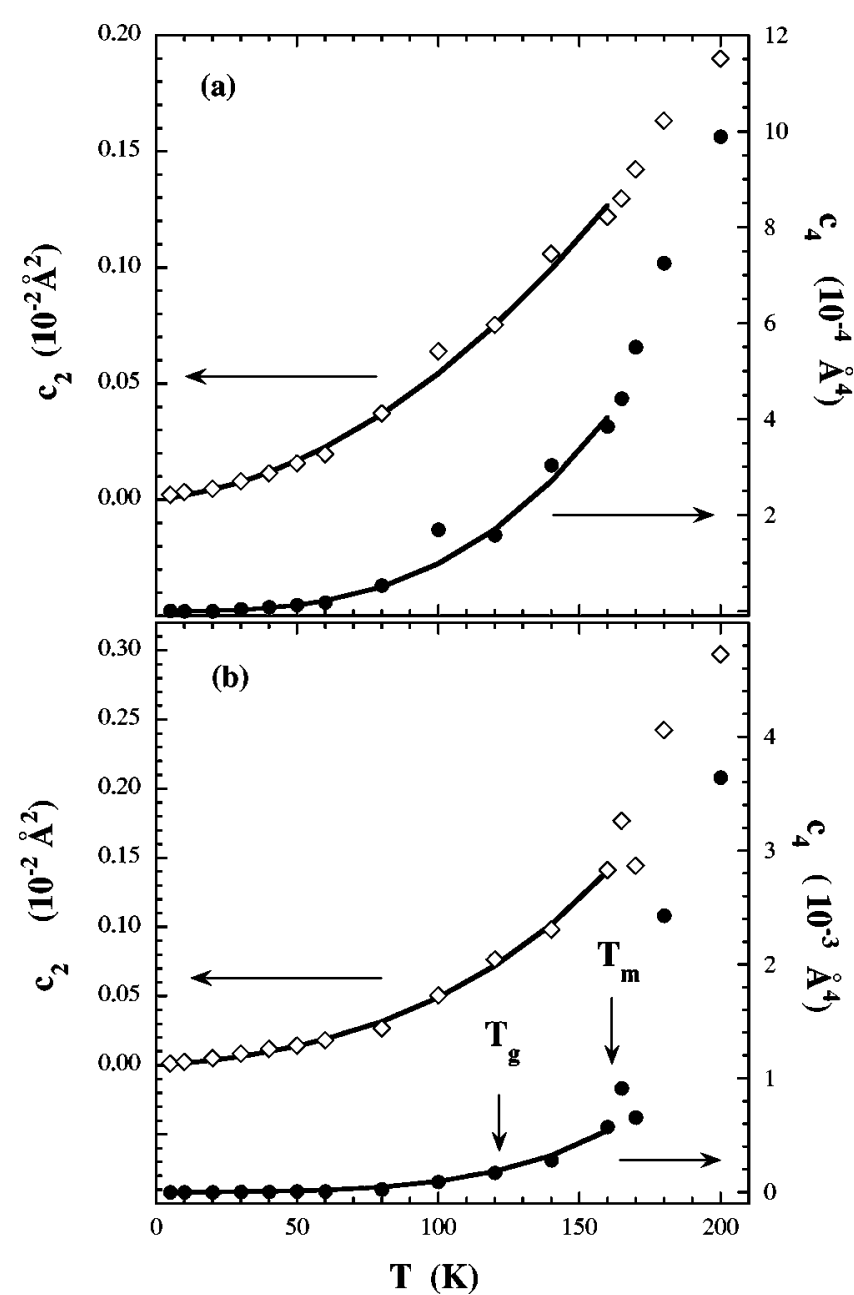

FIG. 7. Dependence with temperature of the quadratic $\left(c_{2}\right.$, open symbols) and quartic ( $c_{4}$, filled symbols) coefficients needed to account for the wave-vector dependence of $2 W(Q, T)$ as calculated for the molecular centers of mass by means of the computer simulations. Data includes temperatures pertaining to the normal liquid range as well as to partially molten bcc crystals. The upper frame shows simulation data for the disordered crystals and the lower frame shows equivalent data for the glass and liquid. Solid lines are fits to Eqs. (7) and (8), respectively.

for the glass and the crystal, respectively, which are remarkably close to those inferred from the experiment at low temperatures (i.e., 0.83 and 0.66 , respectively).

As a first remark let us underline the scant effect felt by $W(T)$ upon crossing both the canonical glass $\rightarrow$ liquid and orientationally disordered $\rightarrow$ rotator-phase crystal transitions, both taking place within $100 \mathrm{~K}-135 \mathrm{~K}$. However, as remarked above, motions indeed are sensitive to the effects of the nonlinear term. To explore in deeper detail this apparent lack of sensitivity to the occurrence of the glass transition we extended the analysis of simulation data up to temperatures comprising the normal-liquid range $\left(T>T_{m}=170 \mathrm{~K}\right)$. Figure 7 displays data already discussed together with those concerning higher temperatures. For the disordered crystals, $c_{2}$ shows a smooth behavior up to the highest temperature while $c_{4}$ shows a somewhat steeper increase with temperature above some $150 \mathrm{~K}$. This is something to be expected by the very nature of the quantity considered in the simulations, that is, since all motions refer to molecular centers of mass we do not expect to see a strong effect due to the onset of molecular rotations upon crossing the orientationally disordered $\rightarrow$ rotator-phase transition. In addition, above $160 \mathrm{~K}$ a slight but noticeable deviation from the behavior followed at lower temperatures by the $c_{4}$ is clearly seen. This is due to partial melting of the bcc lattice, a process that does not reach completion within the time window of our simulations.

In stark contrast with the behavior of the cubic crystals, the $c_{2}$ and $c_{4}$ coefficients for the glass/supercooled liquid exhibit a stronger change upon approaching some $160 \mathrm{~K}$ than those exhibited by the disordered crystals. As a matter of fact, from data pertaining to $c_{4}$ one could find by extrapolation from the high-temperature region a temperature $T^{*}$ about $140 \mathrm{~K}$ that signals the point of the temperature region driven by non-Gaussian dynamics, a feature that also corresponds to the apparent bent in the $A_{n g}^{\max } t^{*}$ plot shown in Fig. 1 . Whether such temperature has any clear meaning is difficult to guess because of the proximity to the upper bound of the glass-transition region within the simulations. Our own estimate for the critical temperature $T_{c}$ of structural arrest corresponding to the schematic mode-coupling prediction made on the basis of muon spectroscopy comes about 118 $\mathrm{K} .{ }^{28}$ The experimental data show, however, a smoother trend that joins the normal liquid regime in a more continuous fashion than does the schematic theoretical prediction. An improved theoretical estimate that includes thermally activated events may well result in a shift of $T_{c}$ towards a temperature closer to $T^{*}$ which will then sign an unequivocal ergodicity-breaking transition.

\section{OUTLOOK AND CONCLUSIONS}

Starting from rather general considerations we have reached at results which for the case of the fully amorphous phases are physically equivalent to the SPM in the sense that the most characteristic features of glassy dynamics are shown to arise from the effect of a large quartic term which gives rise to localized, high-amplitude modes, and that all potential parameters need to be distributed. However, in contrast with the phenomenological nature of the SPM approach we have set to follow a route grounded upon microscopic concepts.

Our route of approaching the glass dynamics has, however, yielded results of interest not included in previous treatments. In fact, the analysis of the CM motions of the disordered crystals, which show all the characteristic fingerprints of glassiness, ${ }^{7-9,25}$ has shown that they can be adequately accounted for by using single-valued potential parameters. In other words, our results emphasize the role played by the nonlinear interactions as main drivers of the glassy dynamics versus the effects brought forward by structural disorder. Support for such a view is lent from a wealth of thermodynamic and dynamic data for the material in consideration $^{7-9,25}$ which evidence the closeness of the glassy dynamics in both glassy phases and above all the recent finding of a large linear term in the low-temperature specific heat which is basically identical for both phases. ${ }^{25} \mathrm{In}$ 
other words, glassy features shown by long-range-ordered arrays having purely orientational disorder are seen to be a consequence of the action of a strong nonlinearity. This suggests that a similar treatment could be applied for systems having orientational or compositional disorder such as the widely studied $\mathrm{KBr}: \mathrm{KCN}$ (Ref. 29) or Ar: $\mathrm{N}_{2}$ alloys. $^{30}$

In conclusion, our study on a sample where the departure from Gaussian behavior can be studied quantitatively has led us to identify some specific features of the effective interaction potential driving the glassy dynamics which appear to be of general validity. The motions of the particles within our simplified model of glassy solids are found to be exceedingly large and highly anisotropic due to the strong cubic and quartic terms of $V(r)$. This serves to provide a framework to understand the wealth of experimental ${ }^{10-14}$ and computer simulation ${ }^{16,17}$ results.

Our main limitation stems from the analysis which has been carried out in terms of the dynamics of molecular centers of mass only. Extending the results beyond those concerning molecular centers of mass to include orientational motions is an obvious next step to take.

\section{ACKNOWLEDGMENTS}

This work was supported in part by DGICYT (Spain) Grant No. MAT2002-04540-C05 and by the Division of Material Sciences, Office of Basic Energy Sciences, U.S. Department of Energy under Contract No. W-31-109-ENG-38. F.J.B. wishes to acknowledge IPNS Division of ANL for financial support.
${ }^{1}$ G.K. White, Phys. Rev. Lett. 34, 204 (1975); Cryogenics 16, 487 (1976); D.A. Ackerman, A.C. Anderson, E.J. Cotts, J.N. Dobbs, W.M. MacDonald, and F.J. Walker, Phys. Rev. B 29, 966 (1984); Y.M. Galperin, V.L. Gurevich, and D.A. Parshin, ibid. 32, 6873 (1985); A. Fujita, T. Suzuki, N. Kataoka, and K. Fukamichi, ibid. 50, 6199 (1994); G. Dalba, P. Fornasini, F. Rocca, and F. Monti, J. Non-Cryst. Solids 293-295, 93 (2001).

${ }^{2}$ A. Würger, From Coherent Tunneling to Relaxation: Dissipative Quantum Dynamics of Interacting Defects (Springer, Berlin, 1997), Chap. 6, p. 116.

${ }^{3}$ E.R. Cowley and G.K. Horton, in Dynamical Properties of Solids, edited by G.K. Horton and A.A. Maradudin (North Holland, Amsterdam, 1995), Vol. 7; D. Acocella, G.K. Horton, and E.R. Cowley, Phys. Rev. Lett. 74, 4887 (1995); Phys. Rev. B 51, 11406 (1995); 61, 8753 (2000); R.C. Shukla and E.R. Cowley, ibid. 31, 372 (1985).

${ }^{4}$ H.E. Fischer, F.J. Bermejo, G.J. Cuello, M.T. Fernandez-Diaz, J. Dawidowski, M.A. Gonzlez, H. Schober, and M. Jimenez-Ruiz, Phys. Rev. Lett. 82, 1193 (1999).

${ }^{5}$ M.A. Gonzalez, E. Enciso, F.J. Bermejo, and M. Bee, Phys. Rev. B 61, 6654 (2000).

${ }^{6}$ R. Fayos, F.J. Bermejo, J. Dawidowski, H.E. Fischer, and M.A. Gonzalez, Phys. Rev. Lett. 77, 3823 (1996); F.J. Bermejo, A. Criado, R. Fayos, R. Fernandez-Perea, H.E. Fischer, E. Suard, A. Guelylah, and J. Zúniga, Phys. Rev. B 56, 11536 (1997).

${ }^{7}$ A. Criado, M. Jimenez-Ruiz, C. Cabrillo, F.J. Bermejo, M. Grimsditch, H.E. Fischer, S.M. Bennington, and R.S. Eccleston, Phys. Rev. B 61, 8778 (2000).

${ }^{8}$ M.A. Ramos, S. Vieira, F.J. Bermejo, J. Dawidowski, H.E. Fischer, H. Schober, M.A. Gonzlez, C.K. Loong, and D.L. Price, Phys. Rev. Lett. 78, 82 (1997); see also Ref. 7; M. JiménezRuiz, M.A. Gonzalez, F.J. Bermejo, M.A. Miller, N.O. Birge, I. Cendoya, and A. Alegria, Phys. Rev. B 59, 9155 (1999); M.A. Miller, M. Jimenez-Ruiz, F.J. Bermejo, and N.O. Birge, ibid. 57, R13 977 (1998); O. Haida, H. Suga, and S. Seki, J. Chem. Thermodyn. 9, 1133 (1974); C. Talón, C. Taln, M.A. Ramos, S. Vieira, G.J. Cuello, F.J. Bermejo, A. Criado, M.L. Senent, S.M. Bennington, H.E. Fischer, and H. Schober, Phys. Rev. B 58, 745 (1998).

${ }^{9}$ A. Criado, M. Jimenez-Ruiz, C. Cabrillo, F.J. Bermejo, R.
Fernandez-Perea, H.E. Fischer, and F.R. Trouw, Phys. Rev. B 61, 12082 (2000).

${ }^{10}$ H. Sillescu, J. Non-Cryst. Solids 243, 81 (1999); R. Richert, J. Phys.: Condens. Matter 14, R703 (2002); R. Zorn, Phys. Rev. B 55, 6249 (1997); U. Buchenau, C. Pecharroman, R. Zorn, and B. Frick, Phys. Rev. Lett. 77, 659 (1996); T. Kanaya, U. Buchenau, S. Koizumi, I. Tsukushi, and K. Kaji, Phys. Rev. B 61, R6451 (2000); A. Arbe, J. Colmenero, F. Alvarez, M. Monkenbusch, D. Richter, B. Farago, and B. Frick, Phys. Rev. E 67, 051802 (2003); A. Arbe, J. Colmenero, F. Alvarez, M. Monkenbusch, D. Richter, B. Farago, and B. Frick, Phys. Rev. Lett. 89, 245701 (2002).

${ }^{11}$ S.A. Reisberg, X.H. Xiu, M. Wilhelm, H.W. Spiess, and M.D. Ediger, J. Chem. Phys. 114, 7299 (2001).

${ }^{12}$ B. Schiener, R.V. Chamberlin, G. Diezemann, and R. Böhmer, J. Chem. Phys. 107, 7746 (1997).

${ }^{13}$ M. Russina, F. Mezei, R. Lechner, S. Longeville, and B. Urban, Phys. Rev. Lett. 84, 3630 (2000).

${ }^{14}$ L.E. Walther, N.E. Israeloff, E. Vidal Russell, and H. Alvarez Gomariz, Phys. Rev. B 57, R15 112 (1998).

${ }^{15}$ T. Odagaki, J. Matsui, and Y. Hiwatari, Phys. Rev. E 49, 3150 (1994); T. Odagaki and Y. Hiwatari, Phys. Rev. A 43, 1103 (1991); J.J. Jäckle, in Disorder Effects in Relaxational Processes, edited by R. Richert (Springer, Berlin, 1994), p. 233; D. Long and F. Lequeux, Eur. J. Phys. 4, 371 (2001); E. Donth, E. Hempel, and C. Schick, J. Phys.: Condens. Matter 12, L281 (2000); H. Huth, M. Beiner, and E. Donth, Phys. Rev. B 61, 15092 (2000).

${ }^{16}$ B. Doliwa and A. Heuer, Phys. Rev. E 61, 6898 (2000); J. NonCryst. Solids 307-310, 32 (2002).

${ }^{17}$ D. Caprion, J. Matsui, and H.R. Schober, Phys. Rev. Lett. 85, 4293 (2000); R. Fernandez-Perea, F.J. Bermejo, and E. Enciso, Phys. Rev. B 53, 6215 (1996); C. Oligschleger, H.R. Schober, Physica A 201, 391 (1993); K. Vollmar-Lee, W. Kob, K. Binder, and A. Zippelius, J. Chem. Phys. 116, 5158 (2002).

${ }^{18}$ M. Jimenez-Ruiz, A. Criado, F.J. Bermejo, G.J. Cuello, F.R. Trouw, R. Fernandez-Perea, H. Löwen, C. Cabrillo, and H.E. Fischer, J. Phys.: Condens. Matter 14, 1509 (2002).

${ }^{19}$ S.W. Lovesey, Theory of Neutron Scattering from Condensed Matter (Oxford Science Publications, Oxford, 1986), Chap. 4. 
${ }^{20}$ A.A. Maradudin and P.A. Flinn, Phys. Rev. 129, 2529 (1963).

${ }^{21}$ W.L. Jorgensen, D.S. Maxwell, and J. Tirado-Rives, J. Am. Chem. Soc. 117, 5179 (1995).

${ }^{22}$ J. Horbach and W. Kob, Phys. Rev. E 64, 041503 (2001).

${ }^{23}$ B.T.M. Willis, Acta Crystallogr., Sect. A: Cryst. Phys., Diffr., Theor. Gen. Crystallogr. 25, 277 (1969).

${ }^{24}$ F.J. Ignatiev, V.G. Karpov, and M. Klinger, J. Non-Cryst. Solids 55, 307 (1983); U. Buchenau, Yu.M. Galperin, V.L. Gurevich, and H.R. Schober, Phys. Rev. B 43, 5039 (1991); K. Bhattacharya, U. Buchenau, and K.W. Kehr, ibid. 49, 8696 (1994).

${ }^{25}$ C. Talón, M.A. Ramos, and S. Vieira, Phys. Rev. B 66, 012201 (2002).

${ }^{26}$ J. Dawidowski, F.J. Bermejo, and J.R. Granada, Phys. Rev. B 58,
706 (1998).

${ }^{27}$ C. Cabrillo, F.J. Bermejo, M. Jimenez-Ruiz, M.T. FernandezDiaz, M.A. Gonzalez, and D. Martin y Marero, Phys. Rev. B 64, 064206 (2002).

${ }^{28}$ C. Cabrillo, F.J. Bermejo, and S.F.J. Cox, Phys. Rev. B 67, 184201 (2003).

${ }^{29}$ E.R. Grannan, M. Randeria, and J.P. Sethna, Phys. Rev. B 41, 7799 (1990).

${ }^{30}$ H.T. Lotz and J.A. Schouten, Phys. Rev. B 64, 024103 (2001), and references therein.

${ }^{31}$ R.M. Lynden-Bell and W. Steele, J. Phys. Chem. 88, 6514 (1984). 